\title{
Spin-gapped incoherent metal with preformed pairing in the doped antiferromagnetic Mott insulator
}

\author{
Ki-Seok Kim and Mun Dae Kim \\ School of Physics, Korea Institute for Advanced Study, Seoul 130-012, Korea
}

(Dated: July 31, 2018)

\begin{abstract}
We investigate how the antiferromagnetic Mott insulator evolves into the d-wave BCS superconductor through hole doping. Allowing spin fluctuations in the strong coupling approach, we find a spin-gapped incoherent metal with preformed pairing as an intermediate phase between the antiferromagnetic Mott insulator and d-wave superconductor. This non-Fermi liquid metal is identified with an infrared stable fixed point in the spin-decomposition gauge theory, analogous to the spin liquid insulator in the slave-boson gauge theory. We consider the single particle spectrum and dynamical spin susceptibility in the anomalous metallic phase, and discuss physical implications.
\end{abstract}

PACS numbers: 71.10.Hf, 71.10.Fd, 71.27.+a, 75.10.-b

\section{INTRODUCTION}

The problem of doped Mott insulators has been one of the central interests in modern condensed matter physics. In particular, the route or mechanism from the parent Mott insulating state to the superconducting phase lies at the heart of the research in strongly correlated electrons. Such a route depends on the nature of the parent Mott insulating phase. Generally speaking, the Mott insulator can be characterized based on its symmetry breaking patterns.[1] Usually, it exhibits symmetry breaking associated with spin rotations or lattice translations, thus causing possible long-range orders. However, when frustration effects are strong enough to kill such orders, the resulting Mott insulator is symmetric, called a spin liquid Mott insulator.[2] Doping to the symmetry-broken and symmetric Mott insulators would result in different routes to superconductivity.

There exist analytical frameworks appropriate to each doped Mott insulator. The doped spin liquid Mott insulator can be described by the slave-boson representation of the t-J model[2] while the doped antiferromagnetic Mott insulator can be captured by its slave-fermion description.[3-5] One of the translationally symmetrybroken insulators may be described by the bond-operator formalism. [6] Our main interest in this paper is doping to the antiferromagnetic Mott insulator, and seeing the emergence of superconductivity from the doped antiferromagnetic Mott insulator. In this respect it seems natural to adopt the slave-fermion representation. Unfortunately, it is believed that the slave-fermion framework does not give rise to the superconductivity naturally. In the next section we review the slave-fermion representation of the t-J model, comparing with the slave-boson approach and discuss the reason why it is not easy to obtain superconductivity in the slave-fermion description. In this study we employ the $\mathrm{CP}^{1}$ spin-decomposition approach[7] which allows superconductivity. The $\mathrm{CP}^{1}$ representation follows the same philosophy as the slavefermion description in the physical point of view.

We have two symmetry breaking phases at both sides of doping, that is, antiferromagnetic Mott insulator at half filling and d-wave superconductor at large doping. The antiferromagnetic Mott insulator is described by the $\mathrm{O}(3)$ nonlinear $\sigma$ model, an effective field theory of the antiferromagnetic Heisenberg model for spin dynamics. Here charge dynamics is almost frozen, thus safely ignored in the low energy limit.[7] On the other hand, the superconducting phase follows the BCS-type approach with d-wave pairing. We show that the $\mathrm{CP}^{1}$ decomposition approach recovers such known theoretical limits naturally.

The main object of this paper is to find the route connecting these well-known symmetry-breaking phases. When holes are doped into the antiferromagnetic Mott insulator, charge fluctuations would be gapless. Then, metallic physics may arise in the absence of disorder, if superconductivity is not taken into account. Actually, numerical simulations based on the t-J model have shown metallic properties.[8] An interesting question is whether such an intermediate metallic phase will survive or not when superconductivity is allowed. The metallic phase may be unstable to disappear. Then, there will be a coexisting phase of antiferromagnetism and superconductivity or the first order transition between them. However, in this paper we show that the metallic state exists indeed as an intermediate phase between the symmetry breaking phases. This kind of metallic phase has been recently argued to appear in the slave-fermion framework. [5] In the $\mathrm{CP}^{1}$ framework we find that the metallic phase turns into a d-wave superconducting state as holes are doped further. We discuss the nature of this metallic phase and find that such a phase is identified with a non-Fermi liquid metal.

Starting from the BCS-HF (Hartree-Fock) model [Eq. (6)], we derive an effective theory: the $\mathrm{CP}^{1}$ representation of the $\mathrm{O}(3)$ nonlinear $\sigma$ model for spin dynamics and the BCS-HF theory for charge dynamics, coupled via $\mathrm{U}(1)$ spin-gauge fluctuations [Eq. (14)]. Here $\mathrm{CP}^{1}$ spin-gauge fluctuations play the role of pairing fluctuations in the pairing term of the fermion sector. As a result, we find a spin-gapped incoherent metal with preformed pairing excitations as an intermediate phase be- 
tween the antiferromagnetic Mott insulator and d-wave superconductor. In the last section we argue that the spin-gapped incoherent metal with preformed pairing is analogous to the spin-gapped "superconducting" state in one dimension.[3] The present study not only generalizes the one dimensional work of Shankar[3] into two dimensions, but also extends the previous studies[4] into an incoherent regime where spin-boson excitations are gapped.

\section{REVIEW OF THE SLAVE-FERMION APPROACH}

To clarify the connection between the present approach and slave-fermion description, it is necessary to review the slave-fermion representation of the t-J model. In addition, to understand the reason why superconductivity does not arise naturally in the slave-fermion approach, we compare the slave-fermion representation of the t-J model with the slave-boson framework. Consider the t-J Hamiltonian

$$
H=-t \sum_{\langle i j\rangle \sigma}\left(c_{i \sigma}^{\dagger} c_{j \sigma}+H . c .\right)+J \sum_{\langle i j\rangle}\left(\vec{S}_{i} \cdot \vec{S}_{j}-\frac{1}{4} n_{i} n_{j}\right)
$$

with the constraint $\sum_{\sigma} c_{i \sigma}^{\dagger} c_{i \sigma} \leq 1$. This inequality constraint is not easy to handle. In order to treat such a constraint a slave-particle decomposition approach can be introduced, since it turns the inequality constraint into an equality one. Depending on the statistics of charge and spin degrees of freedom, one can decompose the electron operator according to the following ways,

$$
\begin{array}{ll}
c_{i \sigma}=b_{i}^{\dagger} f_{i \sigma}, & b_{i}^{\dagger} b_{i}+\sum_{\sigma} f_{i \sigma}^{\dagger} f_{i \sigma}=1, \\
c_{i \sigma}=\psi_{i}^{\dagger} b_{i \sigma}, & \psi_{i}^{\dagger} \psi_{i}+\sum_{\sigma} b_{i \sigma}^{\dagger} b_{i \sigma}=1 .
\end{array}
$$

Here the first line shows the slave-boson representation with the bosonic charge and fermionic spin, and the second line the slave-fermion one with the fermionic charge and bosonic spin.

In each representation the Heisenberg term can be expressed as

$$
\begin{aligned}
& J \sum_{\langle i j\rangle}\left(\vec{S}_{i} \cdot \vec{S}_{j}-\frac{1}{4} n_{i} n_{j}\right)=-\frac{J}{2} \sum_{\langle i j\rangle} \hat{\Delta}_{i j}^{\dagger} \hat{\Delta}_{i j} \\
& \text { slave-boson : } \hat{\Delta}_{i j}=\sum_{\sigma \sigma^{\prime}} \epsilon_{\sigma \sigma^{\prime}} f_{i \sigma} f_{j \sigma^{\prime}}, \\
& \text { slave-fermion : } \hat{\Delta}_{i j}=\sum_{\sigma \sigma^{\prime}} \epsilon_{\sigma \sigma^{\prime}} b_{i \sigma} b_{j \sigma^{\prime}}
\end{aligned}
$$

for the pairing channel. Inserting each decomposition representation into the t-J model with Eq. (3), and performing the Hubbard-Stratonovich transformation for the exchange hopping and Heisenberg pairing channels, one can find each effective Lagrangian

$$
\begin{aligned}
& L_{S B}=\sum_{i} b_{i}^{\dagger} \partial_{\tau} b_{i}-t \sum_{\langle i j\rangle}\left(b_{i}^{\dagger} \chi_{i j}^{b} b_{j}+H . c .\right) \\
& +\sum_{i \sigma} f_{i \sigma}^{\dagger}\left(\partial_{\tau}-\mu\right) f_{i \sigma}-t \sum_{\langle i j\rangle \sigma}\left(f_{i \sigma}^{\dagger} \chi_{i j}^{f} f_{j \sigma}+H . c .\right) \\
& -\sum_{\langle i j\rangle \sigma \sigma^{\prime}}\left(\Delta_{i j}^{\dagger} \epsilon_{\sigma \sigma^{\prime}} f_{i \sigma} f_{j \sigma^{\prime}}+H . c .\right) \\
& +i \sum_{i} \lambda_{i}\left(b_{i}^{\dagger} b_{i}+\sum_{\sigma} f_{i \sigma}^{\dagger} f_{i \sigma}-1\right) \\
& +t \sum_{i}\left(\chi_{i j}^{b} \chi_{i j}^{f}+\text { h.c. }\right)+\frac{1}{2 J} \sum_{\langle i j\rangle}\left|\Delta_{i j}\right|^{2}, \\
& L_{S F}=\sum_{i} \psi_{i}^{\dagger} \partial_{\tau} \psi_{i}+t \sum_{\langle i j\rangle}\left(\psi_{i}^{\dagger} \chi_{i j}^{\psi} \psi_{j}+H . c .\right) \\
& +\sum_{i \sigma} b_{i \sigma}^{\dagger}\left(\partial_{\tau}-\mu\right) b_{i \sigma}-t \sum_{\langle i j\rangle \sigma}\left(b_{i \sigma}^{\dagger} \chi_{i j}^{b} b_{j \sigma}+H . c .\right) \\
& -\sum_{\langle i j\rangle \sigma \sigma^{\prime}}\left(\Delta_{i j}^{\dagger} \epsilon_{\sigma \sigma^{\prime}} b_{i \sigma} b_{j \sigma^{\prime}}+\text { H.c. }\right) \\
& +i \sum_{i} \lambda_{i}\left(\psi_{i}^{\dagger} \psi_{i}+\sum_{\sigma} b_{i \sigma}^{\dagger} b_{i \sigma}-1\right) \\
& +t \sum_{i}\left(\chi_{i j}^{\psi} \chi_{i j}^{b}+\text { h.c. }\right)+\frac{1}{2 J} \sum_{\langle i j\rangle}\left|\Delta_{i j}\right|^{2}
\end{aligned}
$$

in the slave-boson and slave-fermion representations, respectively. Here, $\chi_{i j}^{b, f, \psi}$ is the effective hopping parameter and $\Delta_{i j}$ is the pairing order parameter, both of which will be determined self-consistently. $\lambda_{i}$ is a Lagrange multiplier field to impose single occupancy constraint associated with each decomposition.

Our interest lies in the region where antiferromagnetic correlations are enhanced, captured by spin-singlet pairing fluctuations $\left(\Delta_{i j} \neq 0\right)$. When charge fluctuations are frozen at half filling, the slave-boson approach gives rise to a spin liquid Mott insulating phase, which is a starting point in the slave-boson context. As holes are doped into the spin liquid phase, bosonic charge degrees of freedom becomes condensed to form electronic Cooper pairs, resulting in superconductivity. In this scenario the finite-temperature pseudogap physics is governed by the spin liquid physics for spin fluctuations. On the other hand, the slave-fermion approach will give rise to an antiferromagnetic long-range order at half filling via condensation of bosonic spin degrees of freedom, where fermionic charge fluctuations are gapped to be frozen. As holes are doped into the antiferromagnetic Mott insulator, bosonic spin fluctuations can be gapped and fermionic charge excitations will become gapless to show a metallic phase. $[3,5,9]$ Such a spin-gapped metallic state is expected to govern the pseudogap physics in the slave-fermion context.[5] As will be discussed later, the mathematical structures of both theories are nearly identical. The spin liquid Mott insulating phase can be 
identified with an infrared stable fixed point of the effective fermion-gauge Lagrangian with damped gauge fluctuations, where fermions carry spin degrees of freedom. On the other hand, the spin-gapped metallic phase would be characterized by the same fixed point of nearly the same Lagrangian, but fermions carry charge degrees of freedom here. Since we are focusing on doping the antiferromagnetic Mott insulator, the slave-fermion scheme is more appropriate.

However, there is one serious difficulty in the slavefermion approach. It is not easy to obtain superconductivity in the slave-fermion context. In the slave-boson representation condensation of bosonic charge fluctuations causes superconductivity in the presence of spinsinglet pairing excitations, while fermionic charge degrees of freedom cannot be condensed in the slave-fermion representation. One possible way is to introduce pairing fluctuations of fermionic charge degrees of freedom. Unfortunately, such pairing interactions between fermionic charge degrees of freedom do not arise in the naive meanfield approximation. The Heisenberg term can be written in terms of only bosonic spin degrees of freedom, if the slave-fermion constraint is used appropriately. One can show that such pairing interactions can arise from gauge fluctuations, originating from the slave-fermion decomposition. This is certainly possible, but beyond the mean-field approximation. Our objective is to construct an effective self-consistent mean-field theory in the slavefermion scheme. In addition, we are to examine the fate of such an anomalous metallic phase when superconducting instability is allowed. In this paper we show that the spin-gapped metal appears as an intermediate phase between the antiferromagnetic Mott insulator and d-wave superconductor.

\section{FORMULATION}

\section{A. Model Hamiltonian}

In the previous section we have discussed that the slave-fermion approach allows an anomalous spin-gapped metal via doping to the antiferromagnetic Mott insulator, and such a phase is analogous to the spin liquid state in the slave-boson context. In addition, we argued that it is nontrivial to find superconductivity in the saddle-point analysis of the slave-fermion framework due to the statistics of charge degrees of freedom and the absence of pairing interactions in the mean-field level. In this respect it is necessary to find another representation, keeping the slave-fermion scheme. We consider the t-J-U model Hamiltonian[10]

$$
\begin{aligned}
& H=-t \sum_{\langle i j\rangle \sigma}\left(c_{i \sigma}^{\dagger} c_{j \sigma}+H . c .\right) \\
& -J \sum_{\langle i j\rangle} \sum_{\alpha \beta \gamma \delta}\left(\epsilon_{\alpha \beta} c_{i \alpha}^{\dagger} c_{j \beta}^{\dagger}\right)\left(\epsilon_{\gamma \delta} c_{i \gamma} c_{j \delta}\right)+U \sum_{i} n_{i \uparrow} n_{i \downarrow},(5)
\end{aligned}
$$

where the Heisenberg term is expressed as a pairing channel.

One may argue that interaction terms in this model Hamiltonian are redundant since the $\mathrm{J}$ term can be generated from the $\mathrm{t}-\mathrm{U}$ terms via virtual hopping processes. Although this statement is basically correct, actually the mean-field analysis can hardly captures the effects of exchange interactions if one starts from the Hubbard model. One can understand such a model as follows.[10] Starting from the $\mathrm{t}-\mathrm{U}$ model and integrating out high energy degrees of freedom, one would find the exchange interaction term in the intermediate step of the renormalization group analysis. If the on-site repulsion is not infinitely large, one can keep such an interaction in the intermediate level. Actually, the t-J-U model connects the two limiting cases smoothly. In large- $U$ limit this model is reduced to the t-J model while it recovers the $\mathrm{t}-\mathrm{U}$ model in small-J limit. Remember that the slave-fermion approach in the large- $\mathrm{U}$ limit is not appropriate for describing superconductivity.

Performing the Hubbard-Stratonovich transformation for the pairing channel, we obtain the BCS-Hubbard Hamiltonian as an appropriate model for the doped antiferromagnetic Mott insulator[10, 11]

$$
\begin{aligned}
& H=-t \sum_{\langle i j\rangle \sigma}\left(c_{i \sigma}^{\dagger} c_{j \sigma}+H . c .\right)-\sum_{\langle i j\rangle}\left[\Delta_{i j}\left(c_{i \uparrow}^{\dagger} c_{j \downarrow}^{\dagger}-c_{i \downarrow}^{\dagger} c_{j \uparrow}^{\dagger}\right)\right. \\
& +H . c .]+\frac{1}{J} \sum_{\langle i j\rangle}\left|\Delta_{i j}\right|^{2}+U \sum_{i} n_{i \uparrow} n_{i \downarrow}
\end{aligned}
$$

where the competing nature arising from the densityphase uncertainty is introduced; the $\mathrm{J}$ term causes local pairing $\left(\Delta_{i j}\right)$ of electrons while the Hubbard-U term suppresses local charge fluctuations, thus breaking phase coherence of electron pairs.

Decomposing the Hubbard-U term into the charge and spin channels, we obtain the BCS-HF Lagrangian via the

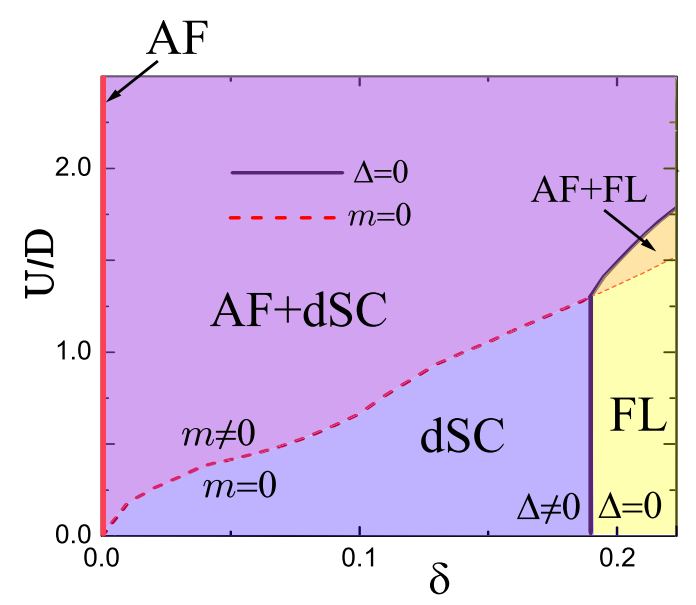

FIG. 1: (Color online) BCS-HF phase diagram with $J / D=$ 0.1 . Separation between the dSC and FL via the straight line is an artifact of the BCS-HF analysis. See the text. 
Hubbard-Stratonovich transformation

$$
\begin{aligned}
& Z=\int D c_{i \sigma} D \varphi_{i} D m_{i} D \vec{\Omega}_{i} D \Delta_{i j} e^{-\int_{0}^{\beta} d \tau L}, \\
& L=\sum_{i \sigma} c_{i \sigma}^{\dagger}\left(\partial_{\tau}-\mu\right) c_{i \sigma}-t \sum_{\langle i j\rangle \sigma}\left(c_{i \sigma}^{\dagger} c_{j \sigma}+H . c .\right) \\
& +\sum_{i}\left(\frac{1}{U} \varphi_{i}^{2}-i \varphi_{i} \sum_{\sigma} c_{i \sigma}^{\dagger} c_{i \sigma}\right) \\
& +\sum_{i}\left(\frac{1}{U} m_{i}^{2}-m_{i} \sum_{\sigma \sigma^{\prime}} c_{i \sigma}^{\dagger}\left(\vec{\Omega}_{i} \cdot \vec{\tau}\right)_{\sigma \sigma^{\prime}} c_{i \sigma^{\prime}}\right) \\
& -\sum_{\langle i j\rangle}\left[\Delta_{i j}\left(c_{i \uparrow}^{\dagger} c_{j \downarrow}^{\dagger}-c_{i \downarrow}^{\dagger} c_{j \uparrow}^{\dagger}\right)+H . c .\right]+\frac{1}{J} \sum_{\langle i j\rangle}\left|\Delta_{i j}\right|^{2}(7)
\end{aligned}
$$

where $\varphi_{i}$ and $m_{i}$ are local charge and spin potentials, respectively.

The saddle-point analysis of Eq. (7) reveals its phase diagram (Fig. 1) with a coexisting phase of antiferromagnetism and d-wave superconductivity $(\mathrm{AF}+\mathrm{dSC})$, a d-wave superconducting state (dSC), an itinerant antiferromagnetic phase $(\mathrm{AF}+\mathrm{FL})$, and a Fermi liquid state $(\mathrm{FL})$ in the plane of $(\delta, U / D)$ with hole concentration $\delta$ and half bandwidth $D$. In this treatment d-wave superconductivity competes with antiferromagnetism. In addition, effects of Hubbard-U interactions can be incorporated only via the antiferromagnetic order parameter. This means that the pairing order parameter does not depend on the Hubbard- $U$ when the antiferromagnetic order parameter vanishes. Considering the fact that the superconducting order parameter vanishes at the point where four phases meet each other, it would also disappear below this point owing to the independence of the pairing order for $\mathrm{U}$, and this separates the superconducting phase from the Fermi liquid state via the straight line which is just a mean-field artifact. This argument seems to be inconsistent with the BCS model analysis. When the antiferromagnetic order disappears, effects of local interactions are not incorporated in this mean-field treatment. Then, the effective Hamiltonian below the red-dotted and red-dashed lines corresponds to the BCS model, which may exhibit superconductivity for all fillings. Actually, however, the pairing interaction strength will decrease effectively as hole concentration increases. The pairing order parameter with $m=0$ is shown in a renormalized mean-field theoretical framework. [12]

In this paper our interest lies in the small doping region where antiferromagnetic correlations would play an important role as discussed in the slave-fermion context. We would like to emphasize that our objective is to show the possible existence of an intermediate paramagnetic metal with gapped spin excitations between the collinear antiferromagnetic and d-wave superconducting phases. The BCS-HF analysis does not take into account charge[13] and spin fluctuations as the heart of Mott physics, particularly, in the low doping region. As a result, the BCS-HF phase diagram does not allow such an intermediate state between the antiferromagnetic Mott insulator and d-wave superconductor. This is inconsistent with the slave-fermion framework which is a strong coupling approach for the doped antiferromagnetic Mott insulator.

\section{B. $\mathbf{C P}^{1}$ representation with pairing fluctuations}

Our objective is to introduce spin fluctuations in the BCS-HF effective theory [Eq. (7)]. A standard approach is to integrate out electron degrees of freedom and obtain an effective action for such spin fluctuations. [14] In this context one can evaluate the self-energy of electrons interacting with spin fluctuations. Although this LandauGinzburg-Wilson-type approach is not self-consistent, one can improve this methodology to be self-consistent performing the Eliashberg-type analysis.[15] Actually, this spin-fluctuation theoretical framework has been applied to quantum phase transitions of interacting itinerant electrons.[16]

However, such a spin-fluctuation theoretical framework is a Fermi-liquid based weak-coupling approach, thus the resulting normal state away from an antiferromagnetic phase is a Fermi liquid state. This is in contrast with the slave-fermion framework of the t-J model, allowing an anomalous metallic state far from the Fermi liquid phase. One optimist may argue that the spin-fluctuation approach can allow such a non-Fermi liquid phase without symmetry breaking if interactions are taken into account more heavily. Unfortunately, this kind of theoretical frameworks have shown only symmetry breaking phases or the Fermi liquid state if symmetric.[17] In particular, the Fermi liquid state was shown to be too stable, even up to two-loop calculations in the renormalization group analysis, [17] to evolve other symmetric metallic phases. In this framework such an anomalous metallic physics can appear only near its quantum critical point. We do not claim that the spin-fluctuation approach is not appropriate for studying the doped Mott insulator. We would like to find the connection with the slave-fermion framework, incorporating spin fluctuations into the BCSHF effective theory. Since our physical motivation lies in finding the anomalous metallic state, claimed to arise in the slave-fermion framework, $[3,5,9]$ we develop a strong coupling approach to allow spin fluctuations in the BCSHF theory.

To incorporate spin fluctuations into the BCS-HF theory $[\mathrm{Eq} .(7)]$ in the context of the strong coupling approach, we resort to the $\mathrm{CP}^{1}$ spin-decomposition $\vec{\Omega}_{i} \cdot \vec{\tau}=$ $U_{i} \tau_{3} U_{i}^{\dagger}$, where $U_{i}=\left(\begin{array}{cc}z_{i \uparrow} & -z_{i \downarrow}^{\dagger} \\ z_{i \downarrow} & z_{i \uparrow}^{\dagger}\end{array}\right)$ is the $\mathrm{SU}(2)$ matrix field with the bosonic spinon $z_{i \sigma} \cdot[18,19]$ Introducing the composite field

$$
\psi_{i \sigma}=U_{i \sigma \sigma^{\prime}}^{\dagger} c_{i \sigma^{\prime}}
$$

in the strong coupling approach,[18, 19] Eq. (7) can be 
expressed as

$$
\begin{aligned}
& Z=\int D \psi_{i \sigma} D z_{i \sigma} D \varphi_{i} D m_{i} D \Delta_{i j} \delta\left(\left|z_{i \sigma}\right|^{2}-1\right) \\
& \exp \left[-\int_{0}^{\beta} d \tau\left\{\frac{1}{U} \sum_{i} \varphi_{i}^{2}+\frac{1}{U} \sum_{i} m_{i}^{2}+\frac{1}{J} \sum_{\langle i j\rangle}\left|\Delta_{i j}\right|^{2}\right.\right. \\
& +\sum_{i \sigma \sigma^{\prime}} \psi_{i \sigma}^{\dagger}\left(\left[\partial_{\tau}-\mu-i \varphi_{i}\right] \delta_{\sigma \sigma^{\prime}}+\left[U_{i}^{\dagger} \partial_{\tau} U_{i}\right]_{\sigma \sigma^{\prime}}\right. \\
& \left.-m_{i} \tau_{\sigma \sigma^{\prime}}^{3}\right) \psi_{i \sigma^{\prime}}-t \sum_{\langle i j\rangle} \sum_{\sigma \sigma^{\prime} \alpha}\left(\psi_{i \sigma}^{\dagger} U_{i \sigma \alpha}^{\dagger} U_{j \alpha \sigma^{\prime}} \psi_{j \sigma^{\prime}}+H . c .\right) \\
& -\sum_{\langle i j\rangle} \Delta_{i j}\left[\left(z_{i \uparrow}^{\dagger} z_{j \downarrow}^{\dagger}-z_{i \downarrow}^{\dagger} z_{j \uparrow}^{\dagger}\right) \psi_{i \uparrow}^{\dagger} \psi_{j \uparrow}^{\dagger}\right. \\
& -\left(z_{i \downarrow} z_{j \uparrow}-z_{i \uparrow} z_{j \downarrow}\right) \psi_{i \downarrow}^{\dagger} \psi_{j \downarrow}^{\dagger}+\left(z_{i \uparrow}^{\dagger} z_{j \uparrow}+z_{i \downarrow}^{\dagger} z_{j \downarrow}\right) \psi_{i \uparrow}^{\dagger} \psi_{j \downarrow}^{\dagger} \\
& \left.\left.\left.-\left(z_{i \downarrow} z_{j \downarrow}^{\dagger}+z_{i \uparrow} z_{j \uparrow}^{\dagger}\right) \psi_{i \downarrow}^{\dagger} \psi_{j \uparrow}^{\dagger}\right]-H . c .\right\}\right] .
\end{aligned}
$$

In this strong coupling representation an antiferromagnetic spin fluctuation $\vec{\Omega}_{i}$ carrying spin quantum number 1 fractionalizes into bosonic spinons $z_{i \sigma}$ with spin $1 / 2$, which seems to occur through the screening of mobile electrons in the antiferromagnetically correlated spin background. The components of $\psi_{i \sigma}$ field are given by $\psi_{i \sigma}=\left(\begin{array}{c}\psi_{i \uparrow} \\ \psi_{i \downarrow}\end{array}\right)=\left(\begin{array}{c}z_{i \uparrow}^{\dagger} c_{i \uparrow}+z_{i \downarrow}^{\dagger} c_{i \downarrow} \\ -z_{i \downarrow} c_{i \uparrow}+z_{i \uparrow} c_{i \downarrow}\end{array}\right)$, which means that mobile electrons in the antiferromagnetically correlated spin background fractionalize into bosonic spinons $U_{i \sigma \sigma^{\prime}}$ and fermionic chargons $\psi_{i \sigma}$, i.e., $c_{i \sigma}=U_{i \sigma \sigma^{\prime}} \psi_{i \sigma^{\prime}}$ in the strong coupling context. An important observation in this representation is that fermion pairing excitations couple to bosonic spin fluctuations, implying that superconductivity is strongly correlated with antiferromagnetism. As discussed in the previous section, it is difficult for the slave-fermion approach to describe such pairing interactions between charge degrees of freedom.

To make the present decomposition scheme natural, it is necessary to understand the present methodology more deeply by comparing this with other well studied ones. A good example is the quantum disordered d-wave superconductivity for high $T_{c}$ cuprates, [20] where the coupling term of $|\Delta| e^{i \phi} c_{\uparrow} c_{\downarrow}$ between Cooper pairs and electrons plays the same role as the exchange coupling term of $\vec{\Omega} \cdot c_{\sigma}^{\dagger} \vec{\tau}_{\sigma \sigma^{\prime}} c_{\sigma^{\prime}}$ between spin fluctuations and electrons. Here, $|\Delta|$ and $\phi$ are the amplitude and phase of Cooper pair fields. To solve this coupling term, several kinds of gauge transformations are introduced. In these decoupling schemes strong phase fluctuations of Cooper pairs, arising from the phase-density uncertainty, screen out charge degrees of freedom of electrons, causing electrically neutral but spinful electrons called "spinons". As a result, the phase factor disappears in the coupling term when it is rewritten in terms of spinons. Instead, this coupling effect appears as current-current interactions of neutral spinons and phase fields of Cooper pairs in the kinetic term of electrons. Depending on the gauge transformations, either $\mathrm{Z}_{2}$ or $\mathrm{U}(1)$ gauge fields are obtained. In this respect the present gauge transformation natu- rally extends the methodology of charge U(1) symmetry in the context of superconductivity to that of spin $\mathrm{SU}(2)$ symmetry in the context of antiferromagnetism.

The "correlated" hopping term can be decomposed in the following way

$$
\begin{aligned}
& \exp \left[-\int_{0}^{\beta} d \tau\left\{\sum_{i \sigma \sigma^{\prime}} \psi_{i \sigma}^{\dagger}\left(\partial_{\tau} \delta_{\sigma \sigma^{\prime}}+\left[U_{i}^{\dagger} \partial_{\tau} U_{i}\right]_{\sigma \sigma^{\prime}}\right) \psi_{i \sigma^{\prime}}\right.\right. \\
& \left.\left.-t \sum_{\langle i j\rangle} \sum_{\sigma \sigma^{\prime} \alpha}\left(\psi_{i \sigma}^{\dagger} U_{i \sigma \alpha}^{\dagger} U_{j \alpha \sigma^{\prime}} \psi_{j \sigma^{\prime}}+\text { H.c. }\right)\right\}\right] \\
& \approx \exp \left[-\left\{-\sum_{i \tau \tau^{\prime}} \sum_{\sigma \sigma^{\prime} \alpha} \psi_{i \tau}^{\dagger \sigma} U_{i \tau}^{\dagger \sigma \alpha} U_{i \tau^{\prime}}^{\alpha \sigma^{\prime}} \psi_{i \tau^{\prime}}^{\sigma^{\prime}}+\sum_{\tau} \sum_{i \sigma} \psi_{i \tau}^{\dagger \sigma} \psi_{i \tau}^{\sigma}\right.\right. \\
& \left.\left.-\frac{t}{J_{\tau}} \sum_{\langle i j\rangle \tau} \sum_{\sigma \sigma^{\prime} \alpha}\left(\psi_{i \tau}^{\dagger \sigma} U_{i \tau}^{\dagger \sigma \alpha} U_{j \tau}^{\alpha \sigma^{\prime}} \psi_{j \tau}^{\sigma^{\prime}}+\text { H.c. }\right)\right\}\right] \\
& =\int D F_{\mu \nu}^{\sigma \sigma^{\prime}} D E_{\mu \nu}^{\sigma \sigma^{\prime}} \exp \left[-\sum_{i \tau \tau^{\prime}}\left\{E_{i \tau \tau^{\prime}}^{\dagger \sigma \sigma^{\prime}} F_{i \tau \tau^{\prime}}^{\sigma^{\prime} \sigma}+H . c .\right.\right. \\
& \left.-U_{i \tau}^{\dagger \sigma \alpha} U_{i \tau^{\prime}}^{\alpha \sigma^{\prime}} F_{i \tau \tau^{\prime}}^{\sigma^{\prime} \sigma}-E_{i \tau \tau^{\prime}}^{\dagger \sigma \sigma^{\prime}} \psi_{i \tau^{\prime}}^{\sigma^{\prime}} \psi_{i \tau}^{\dagger \sigma}-H . c .\right\} \\
& -\sum_{\tau} \sum_{i \sigma} \psi_{i \tau}^{\dagger \sigma} \psi_{i \tau}^{\sigma}-\frac{t}{J_{\tau}} \sum_{\langle i j\rangle \tau}\left\{E_{i j \tau}^{\dagger \sigma \sigma^{\prime}} F_{i j \tau}^{\sigma^{\prime} \sigma}+H . c .\right. \\
& \left.\left.-U_{i \tau}^{\dagger \sigma \alpha} U_{j \tau}^{\alpha \sigma^{\prime}} F_{i j \tau}^{\sigma^{\prime} \sigma}-E_{i j \tau}^{\dagger \sigma \sigma^{\prime}} \psi_{j \tau}^{\sigma^{\prime}} \psi_{i \tau}^{\sigma \dagger}-H . c .\right\}\right],
\end{aligned}
$$

where the time part in the effective hopping term is evaluated in the discrete-time approximation with $J_{\tau}$, an energy scale for discrete time. This kind of approximation has been well adopted in the Monte Carlo simulation, known as the Suzuki-Trotter decomposition.[21] Its detailed derivation can be found in Ref. [19].

The effective hopping parameters can be represented as their amplitudes and phases

$$
\begin{aligned}
& E_{i \tau \tau^{\prime}}^{\dagger \sigma \sigma^{\prime}} \equiv E_{\tau} e^{-i c_{i \tau \tau^{\prime}} \tau_{3 \sigma \sigma^{\prime}}}, \quad F_{i \tau \tau^{\prime}}^{\sigma^{\prime} \sigma} \equiv F_{\tau} e^{i c_{i \tau \tau^{\prime}} \tau_{3 \sigma \sigma^{\prime}}}, \\
& E_{i j \tau}^{\dagger \sigma \sigma^{\prime}} \equiv E_{r} e^{-i c_{i j \tau} \tau_{3 \sigma \sigma^{\prime}}}, \quad F_{i j \tau}^{\sigma^{\prime} \sigma} \equiv F_{r} e^{i c_{i j \tau} \tau_{3 \sigma \sigma^{\prime}}},(11)
\end{aligned}
$$

where the unknown amplitudes are determined selfconsistently in the saddle-point analysis as $E_{\tau}=$ $\left|\left\langle U_{i \tau}^{\dagger \sigma \alpha} U_{i \tau^{\prime}}^{\alpha \sigma^{\prime}}\right\rangle\right|, F_{\tau}=\left|\left\langle\psi_{i \tau^{\prime}}^{\sigma^{\prime}} \psi_{i \tau}^{\dagger \sigma}\right\rangle\right|, E_{r}=\left|\left\langle U_{i \tau}^{\dagger \sigma \alpha} U_{j \tau}^{\alpha \sigma^{\prime}}\right\rangle\right|$, and $F_{r}=\left|\left\langle\psi_{j \tau}^{\sigma^{\prime}} \psi_{i \tau}^{\sigma \dagger}\right\rangle\right|$.

The correlated pairing term can be expressed as

$$
\begin{aligned}
& -\sum_{\langle i j\rangle} \Delta_{i j}\left[\left(z_{i \uparrow}^{\dagger} z_{j \downarrow}^{\dagger}-z_{i \downarrow}^{\dagger} z_{j \uparrow}^{\dagger}\right) \psi_{i \uparrow}^{\dagger} \psi_{j \uparrow}^{\dagger}-\left(z_{i \downarrow} z_{j \uparrow}-z_{i \uparrow} z_{j \downarrow}\right) \psi_{i \downarrow}^{\dagger} \psi_{j \downarrow}^{\dagger}\right. \\
& \left.+\left(z_{i \uparrow}^{\dagger} z_{j \uparrow}+z_{i \downarrow}^{\dagger} z_{j \downarrow}\right) \psi_{i \uparrow}^{\dagger} \psi_{j \downarrow}^{\dagger}-\left(z_{i \downarrow} z_{j \downarrow}^{\dagger}+z_{i \uparrow} z_{j \uparrow}^{\dagger}\right) \psi_{i \downarrow}^{\dagger} \psi_{j \uparrow}^{\dagger}\right]-H . c . \\
& \approx-\sum_{\langle i j\rangle} \Delta_{i j}\left[E_{r}\left(e^{-i c_{i j}} \psi_{i \uparrow}^{\dagger} \psi_{j \downarrow}^{\dagger}-e^{i c_{i j}} \psi_{i \downarrow}^{\dagger} \psi_{j \uparrow}^{\dagger}\right)\right. \\
& \left.-E_{r} \Delta_{i j}^{\psi} \cos \left(\vartheta_{i j}^{\psi}-c_{i j}\right)+\frac{\Delta_{i j}^{\psi}}{2} \sum_{\sigma}\left(z_{i \sigma}^{\dagger} e^{i \vartheta_{i j}^{\psi}} z_{j \sigma}+H . c .\right)\right] \\
& -H . c .
\end{aligned}
$$

in the "saddle-point" approximation, where the meanfield ansatz of $\left\langle\psi_{i \uparrow}^{\dagger} \psi_{j \downarrow}^{\dagger}\right\rangle=\frac{\Delta_{i j}^{\psi}}{2} e^{i \vartheta_{i j}^{\psi}},\left\langle\psi_{i \downarrow}^{\dagger} \psi_{j \uparrow}^{\dagger}\right\rangle=$ 
$-\frac{\Delta_{i j}^{\psi}}{2} e^{-i \vartheta_{i j}^{\psi}},\left\langle\psi_{i \uparrow}^{\dagger} \psi_{j \uparrow}^{\dagger}\right\rangle=0$, and $\left\langle\psi_{i \downarrow}^{\dagger} \psi_{j \downarrow}^{\dagger}\right\rangle=0$ is utilized. The different signs of the phase factor originate from the $\mathrm{U}(1)$ gauge symmetry associated with the $\mathrm{CP}^{1}$ representation [22] while that of the pairing amplitude recovers singlet pairing. It is important to notice that the mean-field ansatz for the pairing sector is consistent with that for the hopping sector.

Performing the continuum approximation for the time part, [19] we obtain the following expression for the effective Lagrangian

$$
\begin{aligned}
& L_{e f f}=\frac{1}{U} \sum_{i}\left(\varphi_{i}^{2}+m_{i}^{2}\right)+\frac{1}{J} \sum_{\langle i j\rangle}\left|\Delta_{i j}\right|^{2}+\mu \sum_{i}(1-\delta) \\
& +\sum_{\langle i j\rangle} E_{r} \Delta_{i j} \Delta_{i j}^{\psi} \cos \left(\vartheta_{i j}^{\psi}-c_{i j}\right)+J_{\tau} \sum_{i}\left(E_{\tau}-1\right) F_{\tau} \\
& +t \sum_{\langle i j\rangle} E_{r} F_{r}+\sum_{i \sigma} \psi_{i \sigma}^{\dagger}\left(E_{\tau}\left[\partial_{\tau}-i \sigma c_{i \tau}\right]-i \varphi_{i}-\sigma m_{i}\right) \psi_{i \sigma} \\
& -\left(\mu+J_{\tau}\left[E_{\tau}-1\right]\right) \sum_{i \sigma} \psi_{i \sigma}^{\dagger} \psi_{i \sigma} \\
& -t E_{r} \sum_{\langle i j\rangle \sigma}\left(\psi_{i \sigma}^{\dagger} e^{-i \sigma c_{i j}} \psi_{j \sigma}+H . c .\right) \\
& -\sum_{\langle i j\rangle} E_{r} \Delta_{i j}\left(e^{-i c_{i j}} \psi_{i \uparrow}^{\dagger} \psi_{j \downarrow}^{\dagger}-e^{i c_{i j}} \psi_{i \downarrow}^{\dagger} \psi_{j \uparrow}^{\dagger}\right)-H . c . \\
& +\frac{F_{\tau}}{J_{\tau}} \sum_{i \sigma}\left|\left(\partial_{\tau}-i c_{i \tau}\right) z_{i \sigma}\right|^{2}-t F_{r} \sum_{\langle i j\rangle \sigma}\left(z_{i \sigma}^{\dagger} e^{i c_{i j}} z_{j \sigma}+H . c .\right) \\
& -\sum_{\langle i j\rangle \sigma} \Delta_{i j} \Delta_{i j}^{\psi} z_{i \sigma}^{\dagger} e^{i \vartheta_{i j}^{\psi}} z_{j \sigma}-H . c .+i \sum_{i} \lambda_{i}\left(\sum_{\sigma}\left|z_{i \sigma}\right|^{2}-1\right),
\end{aligned}
$$

where $\lambda_{i}$ is a Lagrange multiplier field imposing the unimodular constraint. It is important to observe the new energy scale $J_{\tau} / F_{\tau}$ in the boson sector for spin dynamics. It is well known that the energy scale for spin dynamics is different from the Hubbard-U. In this paper we simply assume $F_{\tau} / J_{\tau}=1 / g$ for spin dynamics and $E_{\tau}=1$ for charge dynamics, where $g$ is an effective coupling constant for spin fluctuations, although the full analysis with $F_{\tau}$ and $E_{\tau}$ is possible. This simplification would not alter the phase structure of the present effective theory. It is expected that the $E_{r} \Delta_{i j} \Delta_{i j}^{\psi} \cos \left(\vartheta_{i j}^{\psi}-c_{i j}\right)$ term in the pairing sector [Eq. (12)] is relevant at low energies, allowing us to set $\vartheta_{i j}^{\psi}=c_{i j}+\pi$. Replacing $t F_{r}-\Delta_{i j} \Delta_{i j}^{\psi}$ with $t F_{r}$, the pseudo-fermion pairing order parameter $\Delta_{i j}^{\psi}$ disappears in the effective Lagrangian, and only the electron pairing order parameter $\Delta_{i j}$ appears, consistent with our expectation.

We find the effective Lagrangian for the doped antifer- romagnetic Mott insulator

$$
\begin{aligned}
& L_{e f f}=L_{0}+L_{B C S-H F}+L_{N L \sigma M}, \\
& L_{0}=\frac{1}{U} \sum_{i}\left(\varphi_{i}^{2}+m_{i}^{2}\right)+\frac{1}{J} \sum_{\langle i j\rangle}\left|\Delta_{i j}\right|^{2}+t \sum_{\langle i j\rangle} E_{r} F_{r} \\
& +\mu \sum_{i}(1-\delta), \\
& L_{B C S-H F}=\sum_{i \sigma} \psi_{i \sigma}^{\dagger}\left(\partial_{\tau}-i \sigma c_{i \tau}-\mu-i \varphi_{i}-\sigma m_{i}\right) \psi_{i \sigma} \\
& -t E_{r} \sum_{\langle i j\rangle \sigma}\left(\psi_{i \sigma}^{\dagger} e^{-i \sigma c_{i j}} \psi_{j \sigma}+H . c .\right) \\
& -\sum_{\langle i j\rangle} E_{r} \Delta_{i j}\left(e^{-i c_{i j}} \psi_{i \uparrow}^{\dagger} \psi_{j \downarrow}^{\dagger}-e^{i c_{i j}} \psi_{i \downarrow}^{\dagger} \psi_{j \uparrow}^{\dagger}\right)-H . c ., \\
& L_{N L \sigma M}=\frac{1}{g} \sum_{i \sigma}\left|\left(\partial_{\tau}-i c_{i \tau}\right) z_{i \sigma}\right|^{2} \\
& -t F_{r} \sum_{\langle i j\rangle \sigma}\left(z_{i \sigma}^{\dagger} e^{i c_{i j}} z_{j \sigma}+H . c .\right)+i \sum_{i} \lambda_{i}\left(\sum_{\sigma}\left|z_{i \sigma}\right|^{2}-1\right) .
\end{aligned}
$$

Spin dynamics of the doped antiferromagnetic Mott insulator is governed by the $\mathrm{CP}^{1}$ gauge theory of the nonlinear $\sigma$ model with the renormalized spinon-bandwidth $D F_{r}$. On the other hand, the fermion sector describing charge dynamics coincides with the BCS-HF theory [Eq. (7)] except the renormalized pairing order parameter $E_{r} \Delta_{i j}$ with the renormalized bandwidth $D E_{r}$, ignoring spin-gauge fluctuations $c_{i j}$ in the saddle-point approximation. As a result, we can describe the spin and charge dynamics of the doped antiferromagnetic Mott insulator with three order parameters given by $\left\langle z_{i \sigma}\right\rangle, m_{i}$, and $\Delta_{i j}$.

Since fermion excitations are gapped at half filling, spin fluctuations are only relevant degrees of freedom at low energies, and their dynamics is governed by the $\mathrm{O}(3)$ nonlinear $\sigma$ model. Thus, one theoretical limit of the half-filled case is recovered correctly. Away from half filling, charge fluctuations can be gapless to show metallic properties because the chemical potential shifts from the middle of the BCS-HF gap to the lower band exhibiting finite density of states. In addition, one can find that spin-boson excitations are condensed at moderate values of $U$. These condensed spinons are confined with fermions to form coherent electron excitations, consistent with the conventional BCS-HF theory. Recall that spingauge fluctuations are gapped due to the Anderson-Higgs mechanism. Further hole doping would lead the antiferromagnetic order to vanish while the pairing order survives. The resulting superconducting phase is described by the BCS theory, consistent with the other theoretical limit away from half filling.

An interesting question is whether spinon excitations can be gapped to cause spin-gauge fluctuations gapless in the intermediate doping region between the antiferromagnetic Mott insulator and d-wave superconductor. Since spin-gauge fluctuations play the role of phase fluc- 
tuations of renormalized Cooper pairs $\left(E_{r} \Delta_{i j}\right)$, this intermediate phase would be a spin-gapped metal with preformed pairing. Such an anomalous metal is the primary discovery of the present paper.

\section{Connection with the slave-fermion approach}

It is valuable to find the connection between the slavefermion approach and present theoretical framework. Although its precise connection is not easy to construct, one can understand the relationship qualitatively. It was shown that the slave-fermion Lagrangian at half filling, more precisely the Schwinger-boson theory can recover the $\mathrm{CP}^{1}$ Lagrangian of the nonlinear $\sigma$ model in the longwave length and low energy limits, [23] where the hopping parameter $\chi_{i j}^{b}$ vanishes and the fermion dynamics disappears in the slave-fermion Lagrangian of Eq. (4), that is, $L_{e f f}=\sum_{i \sigma} b_{i \sigma}^{\dagger}\left(\partial_{\tau}+i \lambda_{i}\right) b_{i \sigma}-\sum_{\langle i j\rangle \sigma \sigma^{\prime}}\left(\Delta_{i j}^{\dagger} \epsilon_{\sigma \sigma^{\prime}} b_{i \sigma} b_{j \sigma^{\prime}}+\right.$ $H . c$.) without constant terms. Diagonalizing the effective Hamiltonian, one finds the dispersion relation of boson excitations. Considering such boson excitations around the energy minima and phase fluctuations of singletpairing excitations, one can find the $\mathrm{CP}^{1}$ gauge theory from the above Schwinger-boson Lagrangian in the low energy limit. Here, the $z_{i \sigma}$ field consists of particle-hole linear-combination of the Schwinger-boson field, and the $\mathrm{CP}^{1}$ gauge field arises from the phase field of the singletpairing order parameter.[23]

Hole doping will give rise to a nonzero hopping parameter $\chi_{i j}^{b}$. One can solve such an effective Hamiltonian in the same strategy as the Schwinger-boson case. Actually, one of the present authors is preforming the self-consistent analysis in the presence of fermion excitations. [9, 24] One clear point is that the boson dynamics is relativistic, thus the Klein-Gordon-type Lagrangian will be obtained for low energy dynamics of such boson excitations, corresponding to the $\mathrm{CP}^{1}$ gauge Lagrangian if irrelevant terms are abandoned appropriately.

For fermion dynamics, it is more difficult to find its connection since there are no particle-particle and particle-hole pairing fluctuations in the slave-fermion representation of the t-J model. Even if pairing excitations are neglected in Eq. (14), it is difficult to make the "spin"-dependent chemical potential, $\sigma m$ of Eq. (14) in the slave-fermion Lagrangian of Eq. (4). In addition, there is no spin index in $L_{S F}$ of Eq. (4). One possible way to overcome this inconsistency is to take the low energy limit of Eq. (14). If the long-wave length and low energy limits are considered in Eq. (14), only one flavor of fermions will appear. Then, the resulting lowenergy fermion-gauge Lagrangian with a Fermi surface is expected to be basically the same as that of the slavefermion representation. In section $\mathrm{V}$ we discuss possible low-energy effective Lagrangians in both cases, and argue that the spin-gapped metallic phase where boson excitations are gapped is identified with the same fixed point of the same effective fermion-gauge Lagrangian.
The above discussion tells us that the $\mathrm{CP}^{1}$ decomposition approach of the $\mathrm{HF}$ effective model without pairing fluctuations will share the similar physics with the slavefermion framework of the t-J model, in particular, when bosonic spin fluctuations are gapped. However, there is one difficulty in the present approach. The present decomposition scheme does not have any small parameters, in contrast with the slave-fermion framework where the spin index can be extended as $\sigma=1, \ldots, N$, thus allowing the $1 / N$ expansion. Since the $\mathrm{CP}^{1}$ decomposition can be allowed only in the case of $N=2$, it is not easy to justify its saddle-point analysis against gauge fluctuations. This is the reason why we compare the present framework with the slave-fermion approach, where the mean-field analysis can be justified in the $1 / N$ expansion. There is one more possibility to make the present saddle-point analysis stable against gauge fluctuations. Since gauge fluctuations are dissipative due to the presence of the Fermi surface, strong damping in gauge fluctuations may give rise to the stability of the mean-field analysis.[25] Actually, one of the present authors has discussed that average gauge fluctuations are proportional to $1 / \sigma_{f}$, implying that such fluctuations will be suppressed in the infinite limit of the fermion conductivity $\sigma_{f}$ and allowing the mean-field analysis stable against gauge fluctuations. [25] This important issue is intensively discussed in section V.

\section{MEAN-FIELD ANALYSIS AND PHASE DIAGRAM}

\section{A. Phase diagram}

Taking the mean-field ansatz of antiferromagnetism $\left[m_{i}=(-1)^{i} m\right]$ and d-wave pairing $\left[\Delta_{i j}=\Delta\right.$ with $j=i \pm \hat{x}$ and $\Delta_{i j}=-\Delta$ with $\left.j=i \pm \hat{y}\right]$ with $i \varphi_{i}=\varphi$ and $i \lambda_{i}=\lambda$, we obtain the free energy functional from Eq. (14)

$$
\begin{aligned}
& F_{M F}=\sum_{k}\left(\frac{-\varphi^{2}+m^{2}}{U}+\frac{\Delta^{2}}{2 J}+D E_{r} F_{r}-\mu \delta-\varphi-\lambda\right) \\
& -\frac{1}{\beta} \sum_{\omega_{n}} \sum_{k}^{\prime} \sum_{s, s^{\prime}= \pm} \ln \left(i \omega_{n}-E_{k s s^{\prime}}\right) \\
& +\frac{1}{\beta} \sum_{\nu_{n}} \sum_{k \sigma} \ln \left(\frac{1}{g} \nu_{n}^{2}+F_{r} \epsilon_{k}+\lambda\right) .
\end{aligned}
$$

Here the renormalized fermion spectrum is given by $E_{k \pm \pm}= \pm \sqrt{\left(\sqrt{\left(E_{r} \epsilon_{k}\right)^{2}+m^{2}} \pm|\mu+\varphi|\right)^{2}+\left(E_{r} \Delta_{k}\right)^{2}}$ with $\Delta_{k}=\Delta\left(\cos k_{x}-\cos k_{y}\right)$ and $\epsilon_{k}=-2 t\left(\cos k_{x}+\right.$ $\left.\cos k_{y}\right) \cdot \omega_{n}\left(\nu_{n}\right)$ is the Matzubara frequency for fermions (bosons) with temperature $T \equiv 1 / \beta$.

Minimizing the free energy [Eq. (15)] with respect to $m, \Delta, E_{r}, F_{r}, \lambda, \varphi$, and $\mu$, we obtain the self-consistent mean-field equations. Performing the Matzubara frequency summations and momentum integrals with $\sum_{k}=$ 
$\int_{-D}^{D} d \epsilon D(\epsilon)$ and $D(\epsilon)=1 /(2 D)$ for the boson sector, we

$$
\begin{aligned}
& D F_{r}=\sum_{k}^{\prime}\left[\frac{\left(\sqrt{\left(E_{r} \epsilon_{k}\right)^{2}+m^{2}}+\mu_{r}\right) \frac{E_{r} \epsilon_{k}^{2}}{\sqrt{\left(E_{r} \epsilon_{k}\right)^{2}+m^{2}}}+E_{r} \Delta_{k}^{2}}{\sqrt{\left(\sqrt{\left(E_{r} \epsilon_{k}\right)^{2}+m^{2}}+\mu_{r}\right)^{2}+\left(E_{r} \Delta_{k}\right)^{2}}}\right. \\
& \left.+\frac{\left(\sqrt{\left(E_{r} \epsilon_{k}\right)^{2}+m^{2}}-\mu_{r}\right) \frac{E_{r} \epsilon_{k}^{2}}{\sqrt{\left(E_{r} \epsilon_{k}\right)^{2}+m^{2}}}+E_{r} \Delta_{k}^{2}}{\sqrt{\left(\sqrt{\left(E_{r} \epsilon_{k}\right)^{2}+m^{2}}-\mu_{r}\right)^{2}+\left(E_{r} \Delta_{k}\right)^{2}}}\right], \\
& \frac{2 m}{U}=\sum_{k}^{\prime} \frac{m}{\sqrt{\left(E_{r} \epsilon_{k}\right)^{2}+m^{2}}}
\end{aligned}
$$$$
\left[\frac{\left(\sqrt{\left(E_{r} \epsilon_{k}\right)^{2}+m^{2}}+\mu_{r}\right)}{\sqrt{\left(\sqrt{\left(E_{r} \epsilon_{k}\right)^{2}+m^{2}}+\mu_{r}\right)^{2}+\left(E_{r} \Delta_{k}\right)^{2}}}\right.
$$$$
\left.+\frac{\left(\sqrt{\left(E_{r} \epsilon_{k}\right)^{2}+m^{2}}-\mu_{r}\right)}{\sqrt{\left(\sqrt{\left(E_{r} \epsilon_{k}\right)^{2}+m^{2}}-\mu_{r}\right)^{2}+\left(E_{r} \Delta_{k}\right)^{2}}}\right],
$$$$
\frac{\Delta}{J}=\sum_{k}^{\prime} \Delta\left[\frac{E_{r}^{2}\left(\cos k_{x}-\cos k_{y}\right)^{2}}{\sqrt{\left(\sqrt{\left(E_{r} \epsilon_{k}\right)^{2}+m^{2}}+\mu_{r}\right)^{2}+\left(E_{r} \Delta_{k}\right)^{2}}}\right.
$$$$
\left.+\frac{E_{r}^{2}\left(\cos k_{x}-\cos k_{y}\right)^{2}}{\sqrt{\left(\sqrt{\left(E_{r} \epsilon_{k}\right)^{2}+m^{2}}-\mu_{r}\right)^{2}+\left(E_{r} \Delta_{k}\right)^{2}}}\right] \text {, }
$$$$
\delta=\sum_{k}^{\prime}\left[\frac{\left(\sqrt{\left(E_{r} \epsilon_{k}\right)^{2}+m^{2}}+\mu_{r}\right)}{\sqrt{\left(\sqrt{\left(E_{r} \epsilon_{k}\right)^{2}+m^{2}}+\mu_{r}\right)^{2}+\left(E_{r} \Delta_{k}\right)^{2}}}\right.
$$$$
\left.-\frac{\left(\sqrt{\left(E_{r} \epsilon_{k}\right)^{2}+m^{2}}-\mu_{r}\right)}{\sqrt{\left(\sqrt{\left(E_{r} \epsilon_{k}\right)^{2}+m^{2}}-\mu_{r}\right)^{2}+\left(E_{r} \Delta_{k}\right)^{2}}}\right]
$$

for the fermion part with $\varphi=-\frac{U}{2}(1-\delta)$ and $\mu_{r}=\mu+\varphi$, and

$$
\begin{aligned}
& 1=\frac{\sqrt{\lambda+D F_{r}}-\sqrt{\lambda-D F_{r}}}{\sqrt{1 / g}\left(D F_{r}\right)} \\
& E_{r}=\frac{\left(2 \lambda-D F_{r}\right) \sqrt{\lambda+D F_{r}}-\left(2 \lambda+D F_{r}\right) \sqrt{\lambda-D F_{r}}}{3 \sqrt{1 / g}\left(D F_{r}\right)^{2}}
\end{aligned}
$$

for the spinon sector.

The resulting phase diagram is shown in Fig. 2. The HF phase-boundary characterized by $m=0$ is qualitatively similar with that of Eq. (7) [Fig. 1] although the region of $m \neq 0$ in Fig. 2 is larger than that in the BCS-HF phase diagram, arising from band renormalization $E_{r} D$ to increase the fermion density of states. On the contrary to the BCS-HF phase diagram, we find the region where d-wave pairing order does not exist for

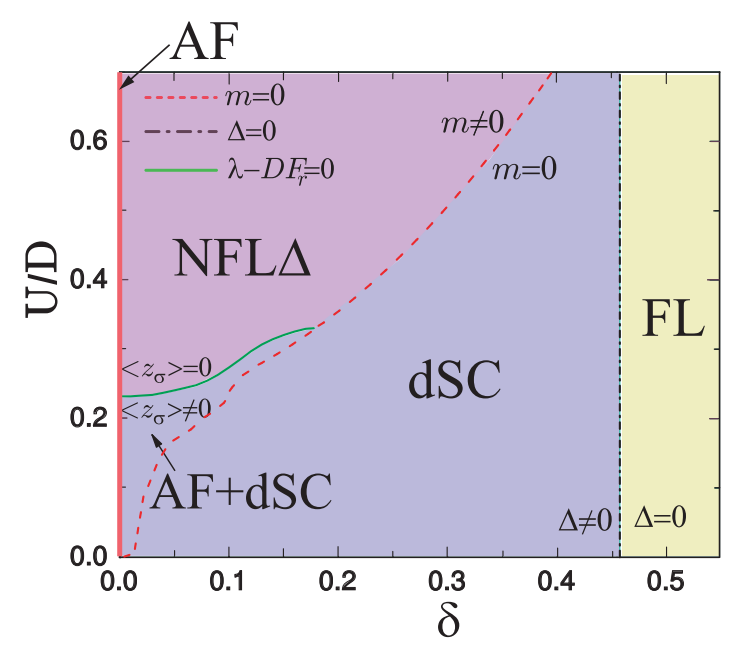

FIG. 2: (Color online) Mean field phase diagram of the spin decomposition theory of Eq. (14) with $J / D=0.7$ and $g=0.42$ at zero temperature. Separation between the dSC and FL via the straight line is an artifact of the BCS-HF analysis for the fermion sector. Introduction of spin fluctuations in the BCS-HF theory alters the AF+dSC phase of the BCS-HF phase diagram into the spin-gapped non-Fermi liquid state with d-wave pairing fluctuations (NFL $\Delta$ ) since such spin fluctuations are gapped in the strong coupling analysis. See the text.

large $U / D$ and low $\delta$. Such a region is not shown in order to clarify the difference between Fig. 2 and Fig. 1, that is, the emergence of a spin-gapped incoherent metal with preformed pairing excitations denoted by NFL $\Delta$, as will be discussed below in more detail. The absence of d-wave pairing in large interaction and small doping originates from the band $\left(E_{r} D\right)$ and pairing $\left(E_{r} \Delta\right)$ renormalization due to spin fluctuations. Actually, the spin-

(16) gapped anomalous metal without pairing fluctuations can be found from the HF Lagrangian without the pairing term, using the same strong coupling approach as the $\mathrm{CP}^{1}$ decomposition.

In Fig. 3 we show doping dependence of BCS-HF order parameters for various $U / D$. The magnetization amplitude scaled by the half bandwidth decreases from its maximum value at half filling as hole concentration increases, exhibiting the second order transition. The dwave pairing order parameter shows an arch-like shape in the parameter range of $U / D$, where it vanishes at half filling due to competition with antiferromagnetism. The black dotted line denotes the point where the magnetization amplitude vanishes, implying that the pairing order parameter does not depend on the Hubbard interaction $U / D$ from this hole concentration, as discussed in the BCS-HF phase diagram [Fig. 1]. By the same reason as the BCS-HF phase diagram the d-wave superconducting phase separates from the Fermi liquid state via the straight line, but this is an artifact of the BCS-HF meanfield analysis for the fermion sector. This doping region is out of interest in the present paper, which we will not 


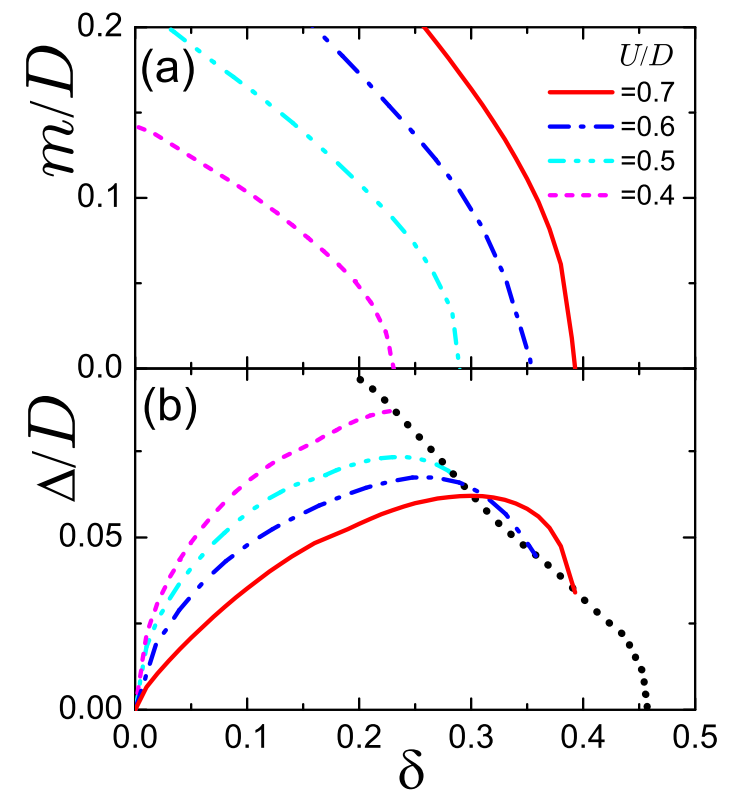

FIG. 3: (Color online) (a) Magnetization amplitude and (b) $\mathrm{d}$-wave pairing gap in the NFL $\Delta$ phase of Fig. 2. The black dotted line shows the pairing gap calculated with $m=0$ in the dSC region.

focus on.

The main point in our spin-decomposition theory is that there is an additional transition line associated with the condensation of spinons. The condensation transition occurs when the boson excitation gap closes, given by $\lambda_{c}-D F_{r_{c}}=0$ in Eq. (17) where the subscript $c$ denotes the quantum critical point. We find the condensation-transition point $(D / g) F_{r c}=2$ with $E_{r c}=1 / 3$ from Eq. (17). Below this transition line the spin-decomposition theory [Eq. (14)] is reduced to the BCS-HF theory [Eq. (7)] owing to the spinon condensation (Higgs phase). Thus, as $\delta$ increases below this line, the phase diagram shows a coexistence region of antiferromagnetism and d-wave superconductivity $(\mathrm{AF}+\mathrm{dSC}$ : $\left.m \neq 0, \Delta \neq 0,\left\langle z_{\sigma}\right\rangle \neq 0\right)$ and d-wave superconducting state (dSC: $m=0, \Delta \neq 0,\left\langle z_{\sigma}\right\rangle \neq 0$ ), perfectly consistent with the BCS-HF phase diagram. Here, the boundary line between $\mathrm{AF}+\mathrm{dSC}$ and $\mathrm{dSC}$ is obtained by extrapolation of $m$ in the NFL $\Delta$ region.[26] Above the transition line, where spin fluctuations are gapped, symmetry-breaking patterns discriminate two non-Fermi liquid (NFL) phases as $U / D$ increases: NFL $\Delta$ with pairing fluctuations $\left(m \neq 0, \Delta \neq 0,\left\langle z_{\sigma}\right\rangle=0\right)$ and NFL $\left(m \neq 0, \Delta=0,\left\langle z_{\sigma}\right\rangle=0\right)$, not shown in Fig. 2.

One cautious person may ask why the AF state is limited to appear at half filling in the case of large $U / D$ although the mean-field phase diagram [Fig. 2] shows such an antiferromagnetic state away from half filling, coexisting with d-wave superconductivity in the case of small $U / D$. This should be regarded as an artifact of the mean-field analysis in the gauge theoretic description. We note that the antiferromagnetic phase is characterized by not only the magnetization amplitude $m$ but also its directional fluctuation $\vec{\Omega}_{i}$. Since the magnetization amplitude is determined by the conventional HF calculation, its nonzero region covers large hole concentration, consistent with the BCS-HF phase diagram [Fig. 1]. However, nonzero $m$ itself does not mean the presence of the antiferromagnetic order, since directional spin-fluctuations may break the magnetic order. When interactions are weak, such directional fluctuations become suppressed. Then, the antiferromagnetic order appears to coexist with the superconducting order, consistent with the BCS-HF result.

As interactions increase, spin fluctuations become strong. Such directional fluctuations are represented as fractionalized boson excitations $z_{\sigma}$ in the strong coupling analysis $\left(\mathrm{CP}^{1}\right.$ decomposition). Even at half filling, such boson excitations can be gapped in the strong coupling case. This is certainly an artifact of the gauge theory approach, which usually occurs in the mean-field calculation. If gauge fluctuations (instanton effects) are taken into account appropriately, confinement should arise at half filling. [27-29] Thus, the antiferromagnetic order will be recovered at half filling as an instanton effect (confinement) when the interaction is large. This also happens in the pure nonlinear $\sigma$ model as the coupling $g$ increases.[30, 31] Such gapped boson excitations should be also confined via gauge interactions.

On the other hand, away from half filling, there emerge gapless fermion excitations. The presence of gapless excitations can give rise to deconfinement, $[25,32-34]$ thus such a disordered phase $\left(\left\langle z_{\sigma}\right\rangle=0 \rightarrow\langle\vec{\Omega}\rangle=0\right)$ may be stabilized. However, the existence of such a deconfinement phase depends on how many flavors of gapless matters there are. $[25,32-34]$ This means that, if hole concentration is small, the density of gapless fermions may not be enough to allow deconfinement.[25, 34] In this case confinement can arise, and such a paramagnetic anomalous metal $\left(m=0\right.$ and $\left.\left\langle z_{\sigma}\right\rangle=0\right)$ becomes unstable in the small $\delta$ and large $\mathrm{U}$ region. As a result, the antiferromagnetic order can persist up to small but finite hole concentration in large U. This antiferromagnetism can be considered as the extension of the antiferromagnetic order at half filling in large $\mathrm{U}$ (arising from confinement in the gauge theory context) to a small doping region. Such a confinement issue will be discussed more deeply in section $\mathrm{V}$. The present mean-field analysis overestimates directional spin-fluctuations since gauge fluctuations are ignored. Introduction of gauge fluctuations has been shown to increase antiferromagnetic correlations.[35] To determine the critical hole concentration where the antiferromagnetic order vanishes is certainly beyond the scope of this paper because it is associated with the confinement issue far beyond the mean-field description.

It is important to understand how the superconducting phase is characterized in the spin-decomposition theory. The BCS superconducting order parameter is not suffi- 
cient to confirm the existence of superconductivity. As shown in the effective Lagrangian Eq. (14), the fermion pairing term consists of $-\sum_{\langle i j\rangle} E_{r} \Delta_{i j}\left(e^{-i c_{i j}} \psi_{i \uparrow}^{\dagger} \psi_{j \downarrow}^{\dagger}-\right.$ $\left.e^{i c_{i j}} \psi_{i \downarrow}^{\dagger} \psi_{j \uparrow}^{\dagger}\right)$. The point is the presence of the phasefluctuation term $e^{-i c_{i j}}$ arising from spin fluctuations. Thus, for superconductivity to be truly realized, not only nonzero $\Delta$ but also $\left\langle e^{-i c_{i j}}\right\rangle \neq 0$ is required, where $\left\langle e^{-i c_{i j}}\right\rangle \neq 0$ can be achieved by boson condensation. Notice that the latter is nothing but the Higgs mechanism since $c_{i j}$ in the phase factor corresponds to the $\mathrm{CP}^{1}$ gauge field. Thus, when such boson excitations are gapped as shown in the mean-field analysis, phase fluctuations of fermion pairs are strong, and the superconducting order does not appear. This is the reason why the non-Fermi liquid phase with preformed pairing fluctuations arises in the strong coupling analysis.

The NFL phase is basically the same as the spingapped metal in the slave-fermion context, where bosonic spin degrees of freedom are gapped, but fermionic charge degrees of freedom are gapless. They are interacting via gauge fluctuations, thus exhibiting anomalous metallic physics. On the other hand, the NFL $\Delta$ phase extends the slave-fermion framework, incorporating pairing fluctuations into the slave-fermion scheme. Thus, the NFL state turns into the NFL $\Delta$ phase in the intermediate $U / D$ above the transition line after superconducting correlations are taken into account. Remember that introduction of superconducting correlations is not trivial in the slave-fermion context since pairing interactions between charge degrees of freedom are not allowed in the naive mean-field scheme.

\section{B. Physical implication}

Both the NFL $\Delta$ and NFL metallic states do not allow coherent electron excitations and spin fluctuations owing to deconfined gapped spinon excitations. Consider the electron Green's function and spin susceptibility

$$
\begin{aligned}
G_{\uparrow \uparrow}^{e l}\left(i j, \tau \tau^{\prime}\right) & \approx g_{\uparrow}^{z}\left(i j, \tau \tau^{\prime}\right) g_{\uparrow}^{\psi}\left(i j, \tau \tau^{\prime}\right) \\
& +g_{\downarrow}^{z}\left(j i, \tau^{\prime} \tau\right) g_{\downarrow}^{\psi}\left(i j, \tau \tau^{\prime}\right), \\
\chi^{z z}\left(i j, \tau \tau^{\prime}\right) & \approx(1-\delta)^{2}\left[g_{\uparrow}^{z}\left(j i, \tau^{\prime} \tau\right) g_{\uparrow}^{z}\left(i j, \tau \tau^{\prime}\right)\right. \\
& \left.+g_{\downarrow}^{z}\left(j i, \tau^{\prime} \tau\right) g_{\downarrow}^{z}\left(i j, \tau \tau^{\prime}\right)\right],
\end{aligned}
$$

where each propagator is expressed as $g_{\uparrow(\downarrow)}^{z}\left(i j, \tau \tau^{\prime}\right)=\left\langle T_{\tau}\left[z_{i \tau \uparrow(\downarrow)} z_{j \tau^{\prime} \uparrow(\downarrow)}^{\dagger}\right]\right\rangle$ and $g_{\uparrow(\downarrow)}^{\psi}\left(i j, \tau \tau^{\prime}\right)=$ $\left\langle T_{\tau}\left[\psi_{i \tau \uparrow(\downarrow)} \psi_{j \tau^{\prime} \uparrow(\downarrow)}^{\dagger}\right]\right\rangle$, respectively. It is important to understand that both response functions consist of convolution integrals. As a result, only "particlehole" continuum spectrum can be observed when spin excitations are fractionalized or deconfined.

Increasing $\delta$ with a fixed $U / D \sim 0.4$ near the spinon condensation-transition in Fig. 2, we pass from the antiferromagnetic Mott insulator to the d-wave superconductor through the spin-gapped incoherent metal with pairing fluctuations. Compared to the high $T_{c}$ phase diagram at zero temperature, the Pseudogap phase may be identified with the NFL $\Delta$, i.e., spin-gapped incoherent metal with preformed pair excitations in the strong coupling analysis of spin fluctuations for the BCS-HF effective theory. The existence of this intermediate nonFermi liquid metal can conceptually explain why offset of superconductivity gives rise to incoherence of elementary excitations. [36, 37] In the deconfined spin-gap phase $(\mathrm{NFL} \Delta)$ both the spin-fluctuation and electron spectra cannot be coherent as shown in the above, and only twoparticle continuum should be observed.

In the spinon-condensed phase with fermion pairing, condensed spinons are confined with fermions to form electron quasiparticles (Higgs phase), thus showing the coherent peak in the single particle spectrum. In the above expression, when boson excitations become condensed, the "two-particle" electron Green's function is reduced to the original one-body Green's function, allowing the coherent peak. In this case such coherent electron excitations also carry spin quantum numbers. This means that the spin susceptibility is expressed as electrons' spin correlations in the superconducting phase. Remember that the spin susceptibility is given by the bosoncorrelation function in the deconfined spin-gap phase $(\mathrm{NFL} \Delta)$ since the spin quantum number is carried by only boson excitations. The point is that if the resonance frequency of spin-fluctuation modes is smaller than the superconducting gap $(2 \Delta)$, such resonance modes can be protected from decaying to electron's particle-hole fluctuations, and sharply defined.[38]

The electron Green's function in the slave-boson representation has the similar expression with that in the spin-decomposition approach except the difference of the quantum number assignment.[2] Thus, boson condensation results in coherent electron excitations in the same way as the above. On the other hand, the spin susceptibility is expressed by fermions' spin correlations in the slave-boson theory. [38] It is important to notice that such fermion fluctuations are not affected by boson condensation severely in the mean-field approximation. In particular, the fermion pairing gap, usually called spin gap, exists in both boson condensed (superconducting) and uncondensed (pseudogap) phases. This protects the magnetic resonance modes from decaying, as discussed above, even in the spin-singlet pairing phase. It may be possible to cure this result by taking into account gauge fluctuations beyond the mean-field approximation. However, at least in the saddle-point approximation, the disappearance of resonance modes in the spin-gap phase is not captured well in the slave-boson theory. One can propose that the superconducting gap closes in the pseudogap phase. In this case the disappearance of such resonance modes can be explained. However, such a proposal has difficulty in explaining the origin of pseudogap. Another mechanism for the pseudogap should be considered in this case. 


\section{BEYOND THE MEAN-FIELD APPROXIMATION}

The spin-decomposition approach has the similar spirit with the spin-fluctuation theory[15] conceptually, because spin fluctuations are taken into account more elaborately. It is also a gauge theory with the same mathematical structure as the slave-boson[2] and slave-fermion theories[3-5]. However, the spin-fluctuation approach is difficult to allow the incoherent metallic phase, which corresponds to a new stable fixed point different from the Fermi liquid phase in the renormalization group sense. A weak coupling approach such as the spin-fluctuation theory is believed to allow either Landau Fermi liquid or conventional symmetry-breaking phases.[17] On the other hand, the effective gauge theory [Eq. (14)], obtained in the strong coupling approach, exhibits an infrared stable fixed point in the presence of gauge interactions (at least without pairing fluctuations), [39] identified as the spin-gapped incoherent metallic phase in the presence of hole doping.

It is necessary to discuss the existence of such a fixed point in more detail, comparing with the slave-boson and slave-fermion contexts. In the slave-boson context the spin liquid insulating phase at half filling where bosonic charge fluctuations are gapped is identified with such a fixed point. Integrating out gapped boson (charge) excitations, one will obtain an effective fermion-gauge action. When the uniform "internal" gauge flux is considered without pairing excitations at half filling, dissipative gauge fluctuations arise to mediate interactions between fermion excitations, thus a non-"relativistic" gauge theory is obtained.[40] If the staggered flux ansatz to allow pairing fluctuations is taken into account at half filling in the $\mathrm{SU}(2)$ formulation context,[2] the "relativistic" $\mathrm{QED}_{3}$ will be obtained, and no damping effects appear in gauge fluctuations. Generically, the non-relativistic fermion-gauge theory can be obtained away from half filling, since hole doping shifts the chemical potential, making a Fermi surface.[40] On the other hand, the spingapped metal where bosonic spin excitations are gapped corresponds to this fixed point in the slave-fermion framework. Integrating out gapped boson (spin) excitations, one would always obtain the non-relativistic fermiongauge action with damped gauge fluctuations away from half filling, since there are no fermion excitations at half filling in the slave-fermion approach of the t-J model. In this respect the effective fermion-gauge theory is generically non-relativistic with dissipative gauge fluctuations for both the slave-boson and slave-fermion frameworks. However, there exists an important difference in the physical point of view; the fermion excitations carry charge quantum numbers in the slave-fermion approach while those do spin quantum numbers in the slave-boson description.

The present gauge theoretic description has exactly the same structure as the slave-particle theoretical framework if $d$-wave pairing excitations are not taken into ac- count. For the time being, we consider the spin-gapped incoherent metal without pairing fluctuations. Integrating out gapped bosonic spin-fluctuations in Eq. (14) without the pairing term, we also find the non-relativistic fermion-gauge action, basically the same as the effective gauge theory of the slave-fermion description in both the physical and mathematical points of view.

The non-relativistic fermion-gauge theory has been argued to have an infrared stable fixed point, [39] where the fixed point value of the internal gauge coupling constant is proportional to $1 / \sigma_{\psi}$ with the fermion conductivity $\sigma_{\psi} \cdot[25,34]$ This is quite reasonable since the fixed point can arise from screening of the internal gauge charge via fermion excitations, and the screening is associated with the fermion conductivity. In the relativistic gauge theory the fixed point charge is proportional to $1 / N$, where $N$ is the fermion flavor number participating in screening of gauge interactions. [32, 33] In this respect the fermion flavor number $N$ in the relativistic theory is analogous to the fermion conductivity in the non-relativistic theory. [25, 34]

An important notorious question is the stability of such an interacting fixed point against instanton excitations which result from compactness of gauge fields. [2729] Although the conclusion is far from consensus, it seems to be possible that when the fermion flavor number or conductivity is large enough to screen the internal gauge charge, instanton excitations can be suppressed, and the interacting fixed point would be stable against confinement.[25, 32-34] Recently, it was argued that the scaling dimension of an instanton insertion operator is proportional to the fermion flavor number $N$ at the conformal invariant fixed point of the relativistic fermiongauge theory. This means that instanton excitations can be irrelevant in the large $N$ limit, expressing the stability of such a fixed point against confinement.[33] Following the similar strategy, one of the present authors critically reinvestigated the stability of the interacting fixed point in the non-relativistic fermion-gauge theory. [25] Since the fermion conductivity in the non-relativistic theory plays the similar role as the flavor number in the relativistic one as mentioned above, it was found that the scaling dimension of the instanton operator is proportional to the fermion conductivity. This implies that instanton excitations would be irrelevant at least in the large conductivity limit corresponding to a good metal. Although we cannot claim the appearance of deconfinement definitely, such an anomalous spin-gapped metal may arise in principle.

Precisely speaking, the interacting fixed point associated with the spin-gapped incoherent metal (NFL) is described by the $z=3$ critical field theory owing to the Landau damping term that results from gapless fermion excitations, where $z$ is the dynamical critical exponent.[19, 25, 41] The effective field theory is well known to cause non-Fermi liquid physics due to scattering with massless gauge fluctuations. The imaginary part of the fermion self-energy is given by $\omega^{2 / 3}$ at the Fermi 
surface, implying that its real part also has the same frequency dependence via the Kramer's Kronig relation, thus giving rise to a non-Fermi liquid behavior.[41] Accordingly, the dc conductivity is proportional to $T^{-5 / 3}$ in three dimensions and $T^{-4 / 3}$ in two dimensions. [40, 42] The coefficient $\gamma$ of the specific heat is proportional to $-\ln T$ in three spatial dimensions and $T^{-1 / 3}$ in two dimensions.[19]

However, there are pairing correlations in NFL $\Delta$. Such pairing fluctuations are expected to be long-rangecorrelated in space but short-range-correlated in time owing to the presence of the Landau damping term in gauge fluctuations. In the presence of pairing fluctuations we don't know how such fluctuations modify the fixed point of NFL without pairing excitations. Since the pairpair correlation function is expected to be singular,[43] these pairing excitations will modify the gauge dynamics, which may change the dynamical critical exponent. Accordingly, this will modify the transport and thermodynamics. The role of pairing fluctuations in the NFL physics needs further investigation.

\section{DISCUSSION AND SUMMARY}

The emergence of the non-Fermi liquid phase in the doped antiferromagnetic Mott insulator can also be supported by its one dimensional analogue. It is well known that low energy physics of the undoped quantum spin chain can be described by the $\mathrm{O}(3)$ non-linear $\sigma$ model with Berry phase.[44] Utilizing the $\mathrm{CP}^{1}$ representation, one can express the non-linear $\sigma$ model in terms of bosonic spinon excitations interacting via compact $\mathrm{U}(1)$ gauge fluctuations in the presence of the Berry phase contribution. Since the Berry phase term is ignorable in the case of integer spin, strong quantum fluctuations originating from low dimensionality lead the integer spin chain to be disordered, causing spinon excitations gapped.[44] Such fractionalized excitations are confined via strong gauge fluctuations, resulting in spin excitons (particle-antiparticle bound states) as elementary excitations. In the case of half-odd integer spin the Berry phase plays a crucial role to cause destructive interference between quantum fluctuations, thus weakening spin fluctuations. Owing to the Berry phase contribution the half-odd integer spin chain is expected to be ordered. But, low dimensionality leads the system to be not ordered but critical, causing the spin-boson excitations gapless.[44] These spinon excitations are deconfined because their critical fluctuations weaken gauge interactions via screening.

When holes are doped into the antiferromagnetic spin chain, Shankar showed that doped holes can be expressed by massless Dirac fermions and these chargefermions interact with the spin-bosons via U(1) gauge fluctuations. [3] The presence of massless Dirac fermions alters the resulting phase completely. Massless Dirac fermions are well known to kill the Berry phase contribu- tion in the bosonization framework. [45] Then, the spinon excitations in the doped half-odd integer spin chain are expected to be massive like those in the undoped integer spin chain. But, these spinons are not confined because gauge fluctuations become massive due to the presence of massless Dirac fermions, thus ignored in the low energy limit. $[3,45]$ In the bosonization framework massless Dirac fermions exhibit superconducting correlations. As a result, the doped antiferromagnetic spin chain is identified with a spin-gapped superconducting phase. Although the mechanism of deconfinement in the $\mathrm{U}(1)$ spin-decomposition gauge theory is completely different from that of the effective theory for the doped spin chain, the spin-gapped incoherent metal with preformed pairing of the $\mathrm{CP}^{1}$ gauge theory is quite analogous to the spin-gapped superconducting state, thus regarded as the high dimensional realization of one dimensional deconfined spin-gapped phase.

The present study is motivated by the possible existence of an anomalous spin-gapped metal in the slavefermion approach of the t-J model. Such a non-Fermi liquid state was argued to be analogous to the spin liquid Mott insulating phase in the slave-boson approach of the t-J model. Although the spin-gapped incoherent metal in the slave-fermion theory is quite appealing, we discussed that it is difficult to incorporate superconducting correlations into the slave-fermion framework due to the fermionic statistics of charge degrees of freedom and the absence of pairing interactions between charge fluctuations in the naive mean-field scheme. In this paper we have developed how to introduce d-wave superconductivity, keeping the slave-fermion scheme.

It is also an important question of this paper how the conventional theoretical framework such as the BCS-HF scheme can give rise to the anomalous metallic phase of the slave-fermion theory. In this paper we found a possible connection between the slave-fermion approach and BCS-HF scheme (beyond), showing how the non-Fermi liquid metal arises from the BCS-HF framework. The spin-fluctuation approach was the first candidate, but it was not adopted in this paper because such a Fermiliquid based weak-coupling approach is difficult to allow the stable non-Fermi liquid phase beyond quantum criticality in the view of its theoretical structure. Instead, we applied the $\mathrm{CP}^{1}$ decomposition scheme as the strong coupling framework. Performing the Hubbard-Stratonovich transformation and appropriate saddle-point approximation, we found an effective gauge theory, quite parallel to the slave-fermion gauge theory. The present $\mathrm{CP}^{1}$ gauge theory allows pairing fluctuations between charge degrees of freedom.

Performing the mean-field analysis, we found the phase diagram of the effective gauge theoretical framework. Effects of spin fluctuations strongly modified the BCS-HF phase diagram [Fig. 1], resulting in the phase diagram of Fig. 2. In particular, the spin-gapped incoherent metallic phase is found when Hubbard-U interactions are beyond a certain critical value. Such a non-Fermi liquid metal 
is certainly expected since it corresponds to that of the slave-fermion theoretical framework. This non-Fermi liquid phase is modified due to pairing correlations of charge degrees of freedom. Actually, we found preformed pairing excitations in the non-Fermi liquid metal near the d-wave superconducting phase.

We have also discussed the stability of such a meanfield phase beyond the mean-field approximation, allowing gauge fluctuations. We claimed that low energy physics of the spin-gapped incoherent metal is described by the non-relativistic fermion-gauge Lagrangian with damped gauge fluctuations, and such an effective field theory gives rise to an infrared stable fixed point. This interacting fixed point identifies the non-Fermi liquid metal beyond the mean-field description. We discussed the stability of such a fixed point against instanton excitations, and argued that the fixed point can be stable against confinement when the fermion conductivity is sufficiently large.

\section{ACKNOWLEDGEMENTS}

K.-S. Kim thanks K. Park for enlightening discussions.
[1] S. Sachdev, Annals Phys. 303226 (2003), and references therein.

[2] P. A. Lee, N. Nagaosa, and X.-G. Wen, Rev. Mod. Phys. 78, 17 (2006), and references therein.

[3] R. Shankar, Phys. Rev. Lett. 63, 203 (1989); R. Shankar, Nucl. phys. B 330, 433 (1990).

[4] B. I. Shraiman and E. D. Siggia, Phys. Rev. Lett. 60, 740 (1988); P. B. Wiegmann, Phys. Rev. Lett. 60, 2445 (1988); S. A. Trugman, Phys. Rev. B 37, 1597 (1988); C. L. Kane, P. A. Lee, and N. Read, Phys. Rev. B 39, 6880 (1989); S. Sachdev, Phys. Rev. B 39, 12232 (1989); X. G. Wen, Phys. Rev. B 39, 7223 (1989); H. J. Schulz, Phys. Rev. Lett. 65, 2462 (1990); P. A. Marchetti, G. Orso, Z. B. Su, and L. Yu, Phys. Rev. B 71, 134510 (2005); C. Brugger, F. Kampfer, M. Moser, M. Pepe, and U.-J. Wiese, Phys. Rev. B 74, 224432 (2006).

[5] R. K. Kaul, A. Kolezhuk, M. Levin, S. Sachdev, and T. Senthil, Phys. Rev. B 75, 235122 (2007); R. K. Kaul, Y. B. Kim, S. Sachdev, and T. Senthil, arXiv:0706.2187 (unpublished).

[6] K. Park and S. Sachdev, Phys. Rev. B 64, 184510 (2001).

[7] A. Auerbach, Interacting Electrons and Quantum Magnetism (Springer-Verlag, New York, 1994).

[8] T.-P. Choy and P. Phillips, Phys. Rev. Lett. 95, 196405 (2005), and references therein.

[9] Performing the saddle-point analysis in the slave-fermion approach of the t-J model, one finds that it is not easy to obtain such a spin-gapped state at zero temperature, where boson excitations are gapped. Actually, considering the uniform flux phase of $\chi_{i j}^{\psi}=\chi_{\psi}$ and $\chi_{i j}^{b}=\chi_{b}$ in the slave-fermion Lagrangian of Eq. (4), only a bosoncondensed phase is found at zero temperature even far away from half filling. One may modify this artificial ordering of the mean-field analysis far away from half filling, introducing frustrating interactions such as next-nearestneighbor or ring-exchange interactions, or competing orders such as the $\pi$-flux phase or superconducting state if possible. In particular, we are investigating the role of $\pi$-flux in the boson condensation, allowing next-nearestneighbor exchange interactions. [24] We expect that effects of both frustration and competition may give rise to the condensation transition in the mean-field analysis of the boson sector. On the other hand, such boson excitations can be gapped at finite temperatures owing to thermal fluctuations, thus finite temperature behaviors may be governed by the spin-gapped metallic physics. [5]
[10] F. C. Zhang, Phys. Rev. Lett. 90, 207002 (2003).

[11] K. Park, Phys. Rev. Lett. 95, 027001 (2005).

[12] F. Yuan, Q. Yuan, and C. S. Ting, Phys. Rev. B 71, 104505 (2005).

[13] Ki-Seok Kim, Phys. Rev. Lett. 97, 136402 (2006); Phys. Rev. B 75, 245105 (2007); Phys. Rev. B 74, 115122 (2006); Phys. Rev. B 73, 235115 (2006).

[14] T. Moriya and J. Kawabata, J. Phys. Soc. Jpn. 34, 639 (1973); T. Moriya and J. Kawabata, J. Phys. Soc. Jpn. 35, 669 (1973); J. A. Hertz, Phys. Rev. B 14, 1165 (1976); A. J. Millis, Phys. Rev. B 48, 7183 (1993).

[15] Ar. Abanov, A. V. Chubukov, and J. Schmalian, condmat/0107421; A. V. Chubukov, D. Pines, and J. Schmalian, cond-mat/0201140.

[16] J. Rech, C. Pepin, and A. V. Chubukov, Phys. Rev. B 74, 195126 (2006).

[17] R. Shankar, Rev. Mod. Phys. 66, 129 (1994).

[18] H.J. Schulz, in The Hubbard Model, edited by D. Baeriswyl (Plenum, New York, 1995); K. Borejsza and N. Dupuis, Phys. Rev. B 69, 085119 (2004); N. Karchev, Phys. Rev. B 5710913 (1998).

[19] Ki-Seok Kim and Mun Dae Kim, Phys. Rev. B 75, 035117 (2007).

[20] T. Senthil and M. P. A. Fisher, Phys. Rev. B 62, 7850 (2000); M. Franz and Z. Tesanovic, Phys. Rev. Lett. 87, 257003 (2001).

[21] M. Suzuki, Prog. of Theor. Phys. 56, 1454 (1976); H. F. Trotter, Proc. Am. Math. Soc. 10, 545 (1959).

[22] The effective Lagrangian will be invariant under the U(1) gauge transformation

$$
\psi_{i \sigma} \rightarrow e^{i \sigma \theta_{i}} \psi_{i \sigma}, \quad z_{i \sigma} \rightarrow e^{-i \theta_{i}} z_{i \sigma},
$$

if the following link variables are transformed as

$$
c_{i j} \rightarrow c_{i j}-\left(\theta_{i}-\theta_{j}\right), \quad \vartheta_{i j}^{\psi} \rightarrow \vartheta_{i j}^{\psi}-\left(\theta_{i}-\theta_{j}\right) .
$$

[23] N. Read and S. Sachdev, Phys. Rev. B 42, 4568 (1990); S. Sachdev and N. Read, Int. J. Mod. Phys. B 5, 219 (1991).

[24] Ki-Seok Kim and Chenglong Jia, in preparation.

[25] Ki-Seok Kim, Phys. Rev. B 72, 245106 (2005).

[26] Considering the case when bosonic spinons are condensed, one should introduce the condensation amplitude $Z=\left|\left\langle z_{i \sigma}\right\rangle\right|^{2}$ in the self-consistent equations for the boson sector. Solving these modified equations, one would find such a transition line between the $\mathrm{AF}+\mathrm{dSC}$ and dSC. In this paper we do not perform such an analysis because 
our objective is to show the existence of the spin-gapped non-Fermi liquid phase. Starting from this solution of the present self-consistent equations [Eqs. (16) and (17)], we can find several instability lines where such an anomalous metal begins to be unstable. This analysis results in the phase diagram of Fig. 2.

[27] A. M. Polyakov, Gauge Fields and Strings (Harwood Academic Publishers, 1987).

[28] E. Fradkin, S. H. Shenker, Phys. Rev. D 19, 3682 (1979).

[29] N. Nagaosa and P. A. Lee, Phys. Rev. B 61, 9166 (2000).

[30] A. M. Tsvelik, Quantum Field Theory in Condensed Matter Physics (Chap. 12, Cambridge University Press, Cambridge, 1995).

[31] N. Nagaosa, Quantum Field Theory in Strongly Correlated Electronic Systems (Springer-Verlag, Berlin, 1999).

[32] T. Senthil, A. Vishwanath, L. Balents, S. Sachdev, and M. P. A. Fisher, Science 303, 1490 (2004); T. Senthil, L. Balents, S. Sachdev, A. Vishwanath, and M. P.A. Fisher, Phys. Rev. B 70, 144407 (2004); H. Kleinert, F. S. Nogueira, and A. Sudbo, Phys. Rev. Lett. 88, 232001 (2002); H. Kleinert, F. S. Nogueira, and A. Sudbo, Nucl. Phys. B 666, 361 (2003); Ki-Seok Kim, Phys. Rev. B 72, 035109 (2005).

[33] M. Hermele, T. Senthil, M. P. A. Fisher, P. A. Lee, N. Nagaosa, and X.-G. Wen, Phys. Rev. B 70, 214437 (2004); F. S. Nogueira and H. Kleinert, Phys. Rev. Lett. 95, 176406 (2005); Ki-Seok Kim, Phys. Rev. B 70, 140405(R) (2004); Ki-Seok Kim, Phys. Rev. B 72, 014406 (2005); Ki-Seok Kim, Phys. Rev. B 72, 214401 (2005)

[34] N. Nagaosa, Phys. Rev. Lett. 71, 4210 (1993).

[35] D. H. Kim and P. A. Lee, Annals Phys. 272, 130 (1999); W. Rantner and X.-G. Wen, Phys. Rev. B 66, 144501 (2002).

[36] A. Damascelli, Z. Hussain, and Z.-X. Shen, Rev. Mod. Phys. 75, 473 (2003).

[37] Y. Sidis, S. Pailhes, B. Keimer, P. Bourges, C. Ulrich, and L.P. Regnault, Phys. Stat. Sol. (b) 241, 1204 (2004).

[38] J. Brinckmann and P. A. Lee, Phys. Rev. B 65, 014502
(2002)

[39] S. Chakravarty, R. E. Norton, and O. F. Syljuasen, Phys. Rev. Lett. 74, 1423 (1995); J. Gan and E. Wong, Phys. Rev. Lett 71, 4226 (1993); C. Nayak and F. Wilczek, Nucl. Phys. B 417, 359 (1994); J. Polchinski, Nucl. Phys. B 422, 617 (1994).

[40] P. A. Lee and N. Nagaosa, Phys. Rev. B 46, 5621 (1992); L. B. Ioffe and G. Kotliar, Phys. Rev. B 42, 10348 (1990).

[41] I. Vekhter and A. V. Chubukov, Phys. Rev. Lett. 93 016405 (2004), and references therein.

[42] One may expect that the response function such as the conductivity is given by the Ioffe-Larkin combination rule [L. B. Ioffe and A. I. Larkin, Phys. Rev. B 39, 8988 (1989)]. However, this is not true owing to the presence of $\sigma= \pm$ in the gauge coupling. Considering the conductivity for example, one finds that it is solely given by the fermion conductivity. Expanding the effective action to the gaussian order for the $\mathrm{U}(1)$ gauge field $c_{\mu}$, the partition function is given in a highly schematic form

$$
\begin{aligned}
& Z=\int D c \exp \left[-\frac{1}{2} \frac{\delta^{2} S_{z}}{\delta c^{2}} c^{2}-\frac{1}{2}\left(\frac{\delta^{2} S_{\psi}}{\delta c^{2}} c^{2}+2 \frac{\delta^{2} S_{\psi}}{\delta c \delta A} c A\right.\right. \\
& \left.\left.+\frac{\delta^{2} S_{\psi}}{\delta A^{2}} A^{2}\right)\right]=Z_{0} \exp \left[-\frac{1}{2} \frac{\delta^{2} S_{\psi}}{\delta A^{2}} A^{2}+\frac{1}{2} \frac{\frac{\delta^{2} S_{\psi}}{\delta a \delta A}}{\frac{\delta^{2} S_{z}}{\delta c^{2}}+\frac{\delta^{2} S_{\psi}}{\delta c^{2}}} A^{2}\right]
\end{aligned}
$$

where $S_{z}\left(S_{\psi}\right)$ is the boson (fermion) action. Differentiating the above effective action by an electromagnetic field $A_{\mu}$ twice, one can obtain the conductivity expression. The presence of $\sigma= \pm$ results in $\delta^{2} S_{\psi} / \delta c \delta A=0$ because correlations between the charge current and spin current are zero. As a result, the conductivity is given by the fermion contribution only.

[43] T. R. Kirkpatrick and D. Belitz, Phys. Rev. Lett. 79, 3042 (1997).

[44] F. D. M. Haldane, J. Appl. Phys. 57, 3359 (1985); I. Affleck, Nucl. Phys. B 265, 409 (1985).

[45] E. Witten, Nucl. phys. B 149, 285 (1979). 